\title{
Genotoxicidad del nifurtimox en diferentes líneas celulares utilizando el ensayo cometa
}

\author{
Laura Fernanda Neira F, Angélica María Vera A, Patricia Escobar*.
}

Centro de Investigaciones en Enfermedades Tropicales (CINTROP), Escuela de Medicina, Departamento de Ciencias Básicas, Universidad Industrial de Santander, Bucaramanga, Colombia.

* Correo electrónico: pescobarwww@yahoo.co.uk.

Fecha de Recepción: 26/02/2016

Fecha de Evaluación: 29/02/2016

Fecha de Solicitud de Correcciones: 01/03/2016

Fecha de Aceptación: 11/03/2016

\section{Resumen}

El nifurtimox es un 5-nitrofurano sintético utilizado en el tratamiento de la enfermedad de Chagas. El objetivo de este estudio fue determinar la toxicidad celular y el daño del ADN causado por el nifurtimox en células Vero, J774, NIH/3T3 y THP-1. Se utilizó la coloración vital con azul tripan y el método colorimétrico MTT para determinar la toxicidad y el ensayo cometa alcalino para determinar el daño al ADN. Los cometas fueron contados en un microscopio de fluorescencia y el porcentaje de daño total del ADN fue calculado y clasificado de 0 (sin daño) a 4 (daño severo). En el ensayo de toxicidad, las células J774 fueron las líneas celulares más sensibles y las células THP-1 las menos sensibles al nifurtimox con valores de $\mathrm{CC}_{50} 34,04$ $138,58 \mu \mathrm{g} / \mathrm{ml}$ y $\mathrm{CC}_{90} 130,58->300 \mu \mathrm{g} / \mathrm{ml}$ de nifurtimox, respectivamente. En el ensayo cometa, el porcentaje de daño total de ADN a $100 \mu \mathrm{g} / \mathrm{ml}$ de nifurtimox fue $79,75 \%, 85,30 \%$ y 10,25\% en células NIH/3T3, J774 y THP-1 respectivamente. En las células Vero el daño del ADN fue del $80 \%$ en células tratadas y no tratadas. El nifurtimox presentó toxicidad y genotoxicidad con actividades que dependieron del tipo de célula y de la concentración del medicamento utilizada. Es importante tomar en cuenta estas diferencias al realizar conclusiones finales de resultados obtenidos utilizando estos ensayos, especialmente el ensayo cometa.
Genotoxiciyt from Nifurtimox in comet assay

Abstract

Nifurtimox is a synthetic 5-nitrofuran used in treating Chagas disease and potentially genotoxic in experimental models. The aim of this study was to determine the cell toxicities and DNA damage caused by nifurtimox on Vero, J774, NIH/3T3 and THP-1 cells. Vital trypan blue staining and MTT colorimetric test for cell toxicities and alkaline comet assay for DNA damage were performed. Comets were counted under fluorescent microscope and the $\%$ of DNA damage was calculated and scored from 0 (no damage) to 4 (high damage). J774 cells was the more sensitive and THP-1 cells the less one with values of $\mathrm{CC}_{50} 34.04-138.58 \mu \mathrm{g} / \mathrm{ml}$ and $\mathrm{CC}_{90} 130.58->300 \mu \mathrm{g} / \mathrm{ml}$. At a dose of $100 \mu \mathrm{g} / \mathrm{ml}$ of nifurtimox, DNA damage was $79.75,85.30 \%$ and $10.25 \%$ on NIH/3T3, J774 and THP-1 cells respectively. Vero cell showed 80\% DNA damage in both treated and untreated cells. Both, cell toxicity and DNA damage were dependent on cell type and drug concentration. It is important to take these variables into account for final conclusions when these types of assays, especially comet assay are performed.

Key words: nifurtimox; cytotoxicity; genotoxicity; comet assay; cell lines.

Palabras clave: nifurtimox; citotoxicidad; genotoxicidad; ensayo cometa; líneas celulares.

\section{Introducción}

El nifurtimox (3-metil-4-(nitrofurfurilidenamina)-tetrahidro-4H-1,4-tiazina-1,1-dioxido, Lampit ${ }^{\oplus}$, Bayer) es un 5-nitrofurano sintético utilizando en el tratamiento de enfermedades producidas por tripanosomas como la enfermedad de Chagas (Tripanosomiasis americana). La enfermedad de Chagas es una enfermedad sistémica compleja que afecta más de 7 millones de personas resultando en aproximadamente 10000 muertes cada año. Representa un problema de Salud Pública en Latinoamérica y es causada por el protozoario flagelado Trypanosoma cruzi (1)(WHO, 2014). Los fármacos nitro-heterocíclicos: nifurtimox (5-nitrofurano) y el benznidazol (2-nitroimidazol) son los medicamentos de elección los cuales son activos contra las diferentes formas de vida del parásito (formas libres e intracelulares) y son eficaces si se administran en las fases agudas de la infección. Los protocolos de tratamiento son largos y dispendiosos presentando reacciones secundarias tales como hipersensibilidad, neuropatías periféricas y alteraciones gastrointestinales en aproximadamente $40 \%$ de los pacientes tratados $(1,2)$.

El mecanismo de acción del nifurtimox se relaciona con su capacidad de reducirse parcialmente y formar radicales de oxigeno (ROS) como el anión superóxido $\left(\mathrm{O}_{2}^{-}\right)$, el peróxido de hidrógeno $\left(\mathrm{H}_{2} \mathrm{O}_{2}\right)$, el radical hidroxilo $\left(\mathrm{OH}^{-}\right)$y nitro-aniones $\left(\mathrm{R}^{-} \mathrm{NO}_{2}^{-}\right)$, que pueden inducir reacciones bioquímicas tales como peroxidación de lípidos, alteración de membranas y degradación del ADN, responsables en gran parte de los efectos secundarios inducidos por el medicamento (2-4).

La genotoxicidad del nifurtimox ha sido reportada desde los años 80 en varios sistemas demostrando ser mutagénico en diversas pruebas (5-9). En modelos experimentales su efecto carcinogénico es discutido. Este fue demostrado en ratones Swiss tratados intraperitonealmente (10) y la toxicidad en el tejido mamario de ratas Sprague-Dawley tratadas intragástricamente (11). Igualmente altas concentraciones del compuesto indujo alteraciones morfo-fisiológicas y/o muerte en embriones de pez cebra (12). Sin embargo contrariamente, tratamiento oral en ratas Wistar no indujo toxicidad crónica ni lesiones neoplásicas en los órganos estudiados (13). En algunos estudios realizados en humanos se han observado aberraciones cromosómicas en linfocitos y presencia de linfomas en niños con enfermedad de Chagas tratados con el medicamento $(14,15)$. 
El ensayo cometa o electroforesis alcalina de células individuales es una técnica utilizada para la detección de lesiones del ADN inducidas por un agente mutagénico. Durante la electroforesis, el ADN intacto permanece en el núcleo pero fragmentos más pequeños con rupturas en sitios álcali lábiles se mueven desde el núcleo hacia afuera asumiendo la forma de un cometa donde la longitud de la cola respecto a la cabeza refleja la número y tamaño de los fragmentos de ADN resultantes (16-20). La ventaja radica en la sensibilidad en detectar los daños del ADN, el número bajo de células requeridas $(<10.000$ células) y su flexibilidad con respecto a tipo de célula eucariótica utilizada (células no-proliferativas y/o proliferativas) $(18,19,21)$. Teóricamente, cualquier célula eucariota puede ser utilizada, sin embargo se recomienda usar líneas celulares bien caracterizadas (21). En las directrices establecidas por la Organización para la Cooperación y el Desarrollo Económico (OECD) en la evaluación de productos químicos, recomiendan el uso de suspensiones de células por ejemplo de hígado y estómago de roedores, sin embargo, la técnica es igualmente aplicable a cualquier tejido o suspensión de células (22). Dada la flexibilidad del ensayo cometa en utilizar diferentes líneas celulares el objetivo del presente trabajo fue evaluar el daño citotóxico y genotóxico inducido por el nifurtimox en diferentes células de mamífero: Vero, J774, NIH/3T3 y THP-1.

\section{Materiales y métodos}

Medicamento y reactivos: Se utilizó el nifurtimox (Bayer ${ }^{\circledR}$, Alemania). El nifurtimox se disolvió en dimetilsulfoxido (DMSO, Carlo Erba, Rodano, MI, Italy) a una concentración final de DMSO menor al $0,5 \%$. Las soluciones de trabajo fueron preparadas en medio de cultivo RPMI-1640 antes de cada ensayo. Las agarosas utilizadas fueron: agarosa con punto de fusión normal y agarosa con bajo punto de fusión (Mo Bio Laboratories Ltda, USA). Los reactivos: Hoesch 33342 (bisBenzimide), azul tripano, MTT (3 -(4. 5 -dimetiltiazol- 2yl)-2. 5difenil-tetrazolio bromuro) y forbol miristato acetato (PMA) fueron obtenidos de Sigma-Aldrich, St. Louis, MO, USA. El cloruro de sodio $(\mathrm{NaCl})$, fosfato de di hidrogeno potasio $\left(\mathrm{KH}_{2} \mathrm{PO}_{4}\right)$, cloruro de potasio $(\mathrm{KCl})$, fosfato di sódico $\left(\mathrm{Na}_{2} \mathrm{HPO}_{4}\right)$, ácido clorhídrico $(\mathrm{HCl})$, hidróxido de sodio $(\mathrm{NaOH})$, tris (hydroximetil) aminometano $\left(\mathrm{NH}_{2} \mathrm{C}\left(\mathrm{CH}_{2} \mathrm{OH}\right)_{3}\right)$ fueron obtenidos de Merck- Darmstadt, Germany. El medio de cultivo RPMI-1640, el suero fetal bovino (SFBi) y la tripsina EDTA fueron obtenidos de Gibco, Canadá.

Cultivos celulares: Las líneas celulares utilizadas fueron: Vero (células epiteliales de riñón de mono verde ATCC CCL-81), THP-1 (monocitos humanos ATCC TIB-202), J774 (monocitos; macrófagos reticulares de ratón BALB/c ATCC TIB-67) y NIH 3T3 (fibroblastos de embrión de ratón NIH/Swiss ATCC CRL-1685, donados amablemente por el Profesor Ricardo Bentes Acevedo de la Universidad de Brasilia). Las células fueron cultivadas en medio de cultivo RPMI-1640 suplementado al $10 \%$ de SFB inactivado con calor (SFBi) a $37^{\circ} \mathrm{C}, 5$ $\% \mathrm{CO}_{2}$ y $95 \%$ de humedad. Las células THP-1 fueron transformadas a su fenotipo adherente con PMA $(40 \mathrm{ng} / \mathrm{ml})$ por 3 días antes para el ensayo de citotoxicidad.

Prueba de citotoxicidad por MTT: Las células a una concentración de $2 \times 10^{5}$ células/ml fueron tratadas con diluciones seriadas de nifurtimox $(11,1-100 \mu \mathrm{g} / \mathrm{ml})$ disuelto en medio de cultivo por 72 $\mathrm{h}$ a $37^{\circ} \mathrm{C}$. Células controles fueron mantenidos sin Nifurtimox. La citotoxicidad se determinó mediante la prueba colorimétrica del MTT (23). Después del tratamiento con nifurtimox las células fueron incubadas con 2,5 mg/ml de MTT por $4 \mathrm{~h}$, los cristales de formazan fueron disueltos con DMSO y la densidad óptica fue determinada por espectrofotometría a $580 \mathrm{~nm}$ usando un lector de microplacas
(Thermo Scientific ${ }^{\mathrm{mx}}$ Multiskan ${ }^{\mathrm{mx}} \mathrm{GO}$ ). El porcentaje de citotoxicidad fue calculado mediante la fórmula: Porcentaje de citotoxicidad $=100$ $\mathrm{x}$ (DO grupo control - DO grupo tratado) / DO grupo control. La actividad fue calculada utilizando análisis de regresión sigmoidal con el programa $\mathrm{MsXl} f \mathrm{it}^{\mathrm{mw}}$ (ID Business Solution, Guildford, UK) y fue expresada como la concentración citotóxica $\left(\mathrm{CC}_{50} \mathrm{y} \mathrm{CC}_{90}\right)$.

Prueba de viabilidad con azul tripan: Las células a $2 \times 10^{5}$ células $/ \mathrm{ml}$ fueron tratadas con diluciones seriadas 1:3 de nifurtimox (11,1-100 $\mu \mathrm{g} / \mathrm{ml}$ ) por $3 \mathrm{~h}$. Posteriormente fueron contadas microscópicamente en cámara de Neubauer utilizando azul de tripan al 0,4\% como colorante vital. Se contaron las células vivas (sin color) y las células muertas (color azul). Los resultados fueron expresados en $\%$ Viabilidad celular $=$ células vivas / células totales $\mathrm{x} 100$.

Ensayo cometa: El ensayo cometa se realizó siguiendo el protocolo descrito por Tice et al., 2000 con algunas modificaciones. Las células (2 x $10^{5}$ células $/ \mathrm{ml}$ ) fueron tratadas con $100 \mu \mathrm{g} / \mathrm{ml}$ de Nifurtimox por $3 \mathrm{~h}$ a $37{ }^{\circ} \mathrm{C} 5 \% \mathrm{CO}_{2} 95 \%$ de humedad. Posteriormente se desprendieron con tripsina-EDTA, se centrifugaron y se re-suspendieron en medio de cultivo. Las células tratadas se mezclaron con agarosa de punto bajo de fusión $0,5 \%(\mathrm{w} / \mathrm{v}) \mathrm{PBS}, \mathrm{pH} 7,4$ at $37^{\circ} \mathrm{C}$ y posteriormente se pipetearon sobre una lámina portaobjetos cubierta con agarosa de punto normal de fusión al 1,5 \% en PBS pH 7,2. Se colocó una laminilla sobre cada una de las láminas y se llevaron a $4{ }^{\circ} \mathrm{C}$ hasta solidificar. Después se retiró la laminilla y las láminas se colocaron en una solución de lisis alcalina $\left(2.5 \mathrm{M} \mathrm{NaCl}, 100 \mathrm{mM} \mathrm{Na} \mathrm{EDTA}_{2}, 10 \mathrm{mM}\right.$ Tris, pH 10.0, $1 \%$ Triton X-100, $10 \%$ DMSO) por $1 \mathrm{~h}$ a $4{ }^{\circ} \mathrm{C}$. Luego se colocaron en una cámara de electroforesis horizontal (Labnet, Miami-USA), se cubrieron con el tampón de electroforesis (10 M $\mathrm{NaOH}, 200 \mathrm{mM} \mathrm{Na} \mathrm{EDTA}_{2} \mathrm{pH}$ 13,0) por 30 min para equilibrarse y luego se realizó la electroforesis a $25 \mathrm{~V}$ y $300 \mathrm{~mA}, 4{ }^{\circ} \mathrm{C}$ por $40 \mathrm{~min}$. Posteriormente las láminas fueron neutralizadas con una solución de tampón Tris (0,4 M Tris, pH 7,5) por $15 \mathrm{~min}$. Las células se fijaron con etanol por $10 \mathrm{~min}$ y se tiñeron con $10 \mu \mathrm{g} / \mathrm{ml}$ Hoechst 33342 por 3 min (24). Se contaron 100 cometas por cada lámina utilizando un microscopio de epifluorescencia (Nikon eclipse E400) filtro UV2A $(\mathrm{Ex}=330-380, \mathrm{DM}=400, \mathrm{BA}=420)$. Se tomaron registros utilizando una cámara fotográfica (Nikon Coolpix P6000, Japan).

Análisis de resultados: En las pruebas de toxicidad se realizaron dos experimentos, se utilizó la prueba de $\mathrm{t}$ de Student y un valor de $\mathrm{P}<$ 0,05 fue considerado estadísticamente significativo.

La medida de la migración del ADN se evaluó mediante puntuación visual de acuerdo a la longitud de la cola del cometa, clasificándolas en cinco categorías (0-4) (19). El número de cometas en cada categoría fue contado en 100 células y el índice de daño (ID) se calculó de acuerdo a la formula propuesto por Jaloszynski et al., 1997. ID = (clase $1 \times 1)+($ clase $2 \times 2)+($ clase $3 \times 3)+($ clase $4 \times 4) /$ total de células analizadas (25).

\section{Resultados}

Citotoxicidad de nifurtimox en las diferentes líneas celulares: El rango de toxicidad al nifurtimox a las $72 \mathrm{~h}$ en términos de $\mathrm{CC}_{50}$ fue de 34,04 $138,58 \mu \mathrm{g} / \mathrm{ml}$ y de $\mathrm{CC}_{90}$ fue de 130,58 - >300 $\mu \mathrm{g} / \mathrm{ml}$ siendo las células J774 las más sensibles y las células THP-1 las menos. Se evidenció una dosis respuesta en la actividad citotóxica del nifurtimox en las líneas evaluadas (Figura 1). El tratamiento con nifurtimox por tres horas no afectó la viabilidad de las células encontrándose valores de viabilidad en más del $80 \%$ en todas las líneas celulares evaluadas (dato no mostrado). 
Figura 1. Citotoxicidad in vitro del nifurtimox en las líneas celulares Vero, THP-1, J774 y NIH/3T3 después de 72 h de exposición a 11,33 y 100 $\mu \mathrm{g} / \mathrm{ml}$ de nifurtimox. El resultado mostrado es el resultado de dos experimentos.

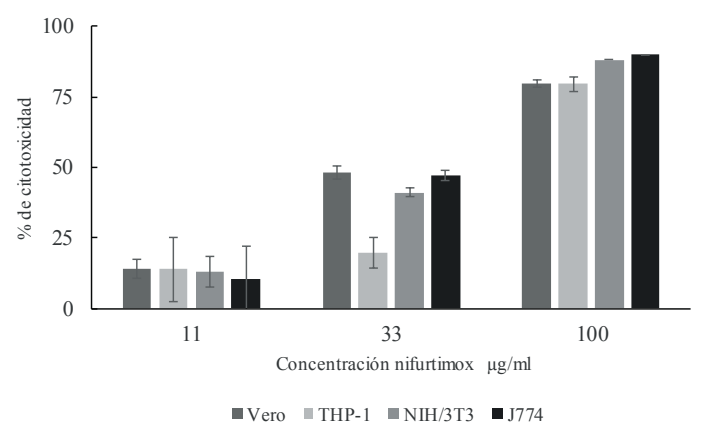

Genotoxicidad al nifurtimox: El daño del ADN en las líneas celulares expuestas a $100 \mu \mathrm{g} / \mathrm{ml}$ de nifurtimox se muestra en la figura 2 y cuadro 1.

Cuadro 1. Porcentajes del daño en el ADN e índice de daño (ID) producido por el nifurtimox en células de mamífero J774, NIH/3T3, Vero y THP-1.

\begin{tabular}{llllllllll}
\hline \multirow{2}{*}{ Línea celular } & \multirow{2}{*}{$\mathrm{NFX} \mu \mathrm{g} / \mathrm{ml}$} & 0 & \multicolumn{1}{c}{ Clase de Daño ADN } & \multicolumn{2}{c}{ ID $\pm \mathrm{DS}$} & \% Daño Total \pm DS \\
\hline \multirow{2}{*}{ J774 } & 0 & 71,67 & 25,67 & 2,00 & 0,33 & 0,33 & $32 \pm 18,1$ & $8 \pm 4,5$ \\
& 100 & 0,00 & 0,00 & 15,00 & 51,00 & 34,00 & $319 \pm 16$ & $79,75 \pm 4,0$ \\
NIH/3T3 & 0 & 62,67 & 34,67 & 1,67 & 0,67 & 0,33 & $41,33 \pm 7,1$ & $10,33 \pm 1,7$ \\
& 100 & 0,00 & 0,00 & 2,33 & 52,67 & 44,67 & $341,33 \pm 8,6$ & $85,33 \pm 2,2$ \\
VERO & 0 & 0,00 & 11,00 & 7,50 & 28,00 & 52,50 & $320 \pm 91$ & $80 \pm 22,9$ \\
& 100 & 0,50 & 4,00 & 22,50 & 34,00 & 43,00 & $323 \pm 74,9$ & $80,75 \pm 18,7$ \\
THP-1 & 0 & 85,50 & 11,50 & 2,50 & 0,50 & 0,00 & $18 \pm 14,1$ & $4,5 \pm 3,5$ \\
& 100 & 62,00 & 35,50 & 2,00 & 0,50 & 0,00 & $41 \pm 33,9$ & $10,25 \pm 8,4$ \\
\hline
\end{tabular}

NFX: Nifurtimox, ID:índice de daño, DS: Desviación estándar.

Figura 2. Genotoxicidad del nifurtimox en diferentes líneas celulares. Las fotografías muestran la presencia de cometas en las diferentes líneas celulares: J774, NIH/3T3, Vero y THP-1 tratadas con $100 \mu \mathrm{g} / \mathrm{ml}$ de nifurtimox (NFX). En la línea superior se muestran los resultados de los controles negativos (células tratadas con medio RPMI-1640 sin suero). Los resultados mostrados corresponden a un experimento representativo de al menos siete ensayos realizados.
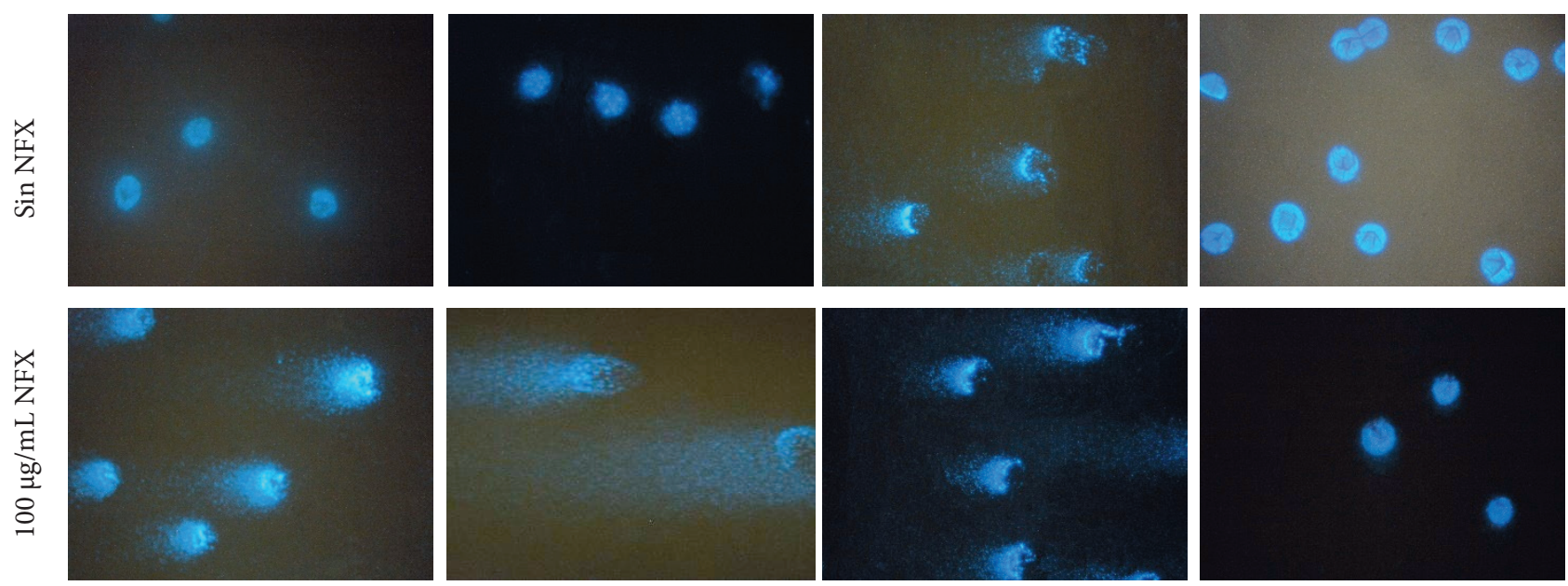

$\mathrm{J} 774$

$\mathrm{NIH} / 3 \mathrm{~T} 3$

Vero

THP-1

El tratamiento con nifurtimox por 3 h presentó daño en el ADN con algunas diferencias importantes entre las células utilizadas. En las células $\mathrm{NIH} / 3 \mathrm{~T} 3$ y las células J774 el porcentaje de daño de ADN fue similar con valores de $85,33 \pm 2,2$ y 79,75 $\pm 4,0 \%$ respectivamente. Sin embargo en las células THP-1 el porcentaje total de daño del ADN fue sólo de $10,25 \pm 8,4 \%$ muy similar al control sin medicamento. Por otro lado, en las células Vero el resultado no fue concluyente ya que se observaron porcentajes elevados de daño del ADN en el control negativo sin nifurtimox $(80 \pm 22,9 \%)$. Experimentos utilizando diferentes concentraciones del nifurtimox fueron realizados encontrándose en las células J774 (Figura 3) y las $\mathrm{NIH} / 3 \mathrm{~T} 3$ un efecto de dosis respuesta. 


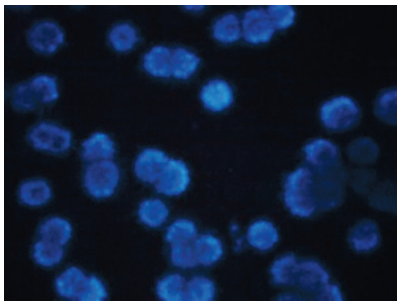

A

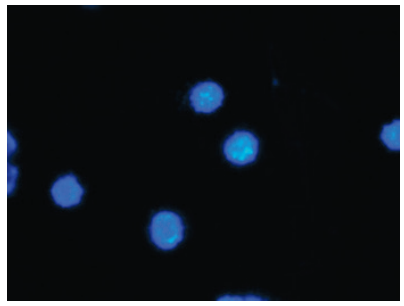

B

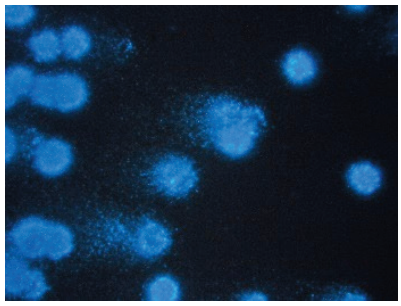

C

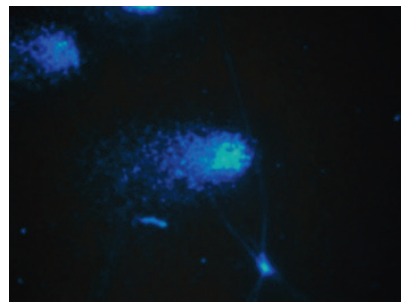

$\mathrm{D}$

Figura 3. Dosis respuesta del nifurtimox en la línea celular J774. A. Control negativo B. nifurtimox $11,1 \mu \mathrm{g} / \mathrm{ml}$ C. nifurtimox $33,3 \mu \mathrm{g} / \mathrm{ml}$ y $\mathbf{D}$. nifurtimox $100 \mu \mathrm{g} / \mathrm{ml}$.

\section{Discusión}

El efecto citotóxico y genotóxico del nifurtimox está siendo reevaluado utilizando varias pruebas con resultados desalentadores tanto para el benznidazol como el nifurtimox sugiriendo que éstos podrían actuar directamente como mutágenos induciendo citotoxicidad y genotoxicidad en linfocitos humanos (4). En el presente trabajo se demostró la actividad citotóxica y genotóxica del nifurtimox en diferentes líneas celulares. Estas actividades dependieron de la concentración del medicamento y del tipo de célula utilizado. La variabilidad en la toxicidad del nifurtimox ha sido demostrada en ensayos in vitro utilizando varios tipos de células de mamífero hospederas del T. cruzi ya que este medicamento es utilizado como medicamentos de referencia en estudios de screening de nuevos compuesto anti-tripanosomas (26-29). Igualmente y dado que el nifurtimox está siendo evaluado como medicamento en el tratamiento de algunos canceres como neuro y meduloblastoma, su actividad antitumoral ha sido demostrada en diferentes tipos de células cancerosas (30). Ensayos in vitro en líneas celulares de neuroblastoma mostraron ligeras diferencias en la toxicidad al nifurtimox siendo más susceptibles las que presentaban mayor producción de ROS (31). Se demostró además que el nifurtimox inducía apoptosis en células de neuroblastoma y en células xenotransplantadas en ratones desnudos (31).

En el presente trabajo, se encontraron diferencias significativas en el daño del ADN a una misma concentración del medicamento, siendo las células NIH/3T3 y las J774 más susceptibles que las THP-1. Esos efectos en el daño del ADN asociado a la línea celular podrían deberse diferencias intrínsecas en las células implicadas en los mecanismos de producción de ROS entre otros. El nifurtimox es un 5-nitrofurano que funciona como una pro-droga la cual tiene que nitroreducirse para ejercer su actividad. De esta manera se producen $\mathrm{R}-\mathrm{NO}_{2}-\mathrm{O}_{2}^{-} \mathrm{y}^{-}$en consecuencia $\mathrm{H}_{2} \mathrm{O}_{2}$ y OH los cuales interactúan con macromoléculas produciendo daño celular $(2,3)$. Su toxicidad tanto en pacientes como en modelos experimentales ha sido relacionada con su biotransformación a nitroaniones y la generación de ROS que conducen a daños en el ADN, lípidos de la membrana celular y otros componentes celulares esenciales (32). Esta reducción que sufre el grupo nitro influye fuertemente en su capacidad mutagénica del nifurtimox.

Las células NIH/3T3 han sido utilizadas ampliamente en la prueba cometa para determinar la genotoxicidad de diferentes compuestos/moléculas. Los niveles basales del daño de ADN encontrados en los estudios fueron semejantes a los encontrados en este estudio observándose una relación $<8$ entre las células tratadas y las no tratadas. Una dosis respuesta en el daño genotóxico se ha observado al tratamiento con hormonas del estrés (33), nanopartículas de óxido de Zn y oxido de Ti $(34,35)$ y un efecto protectivo de extractos de plantas como el de guaraná a la exposición con nitroprusito de sodio $(36,37)$. Por otro lado la caracterización de algunos de los genes implicados en el daño genotóxico (37) y la aprobación por la OECD para ser utilizadas en ensayos de fototoxicidad (20) nos indica que es uno de los mejores modelos de células a utilizar en estos ensayos. Diferente al uso de las NIH/3T3 en la prueba cometa, las células J774 (macrófagos murinos derivados de BALB/c) han sido menos utilizadas $(38,39)$. El hecho que presentaran resultados de daño al ADN comparables con las NIH/3T3, siendo estos resultados bastante reproducibles en los diferentes experimentos, igualmente su facilidad de mantenimiento en condiciones de laboratorio, podría constituir en buen modelo para ser utilizada como células blanco en los ensayos cometa.

En el presente estudio las células THP-1 no diferenciadas fueron las menos susceptibles al daño genotóxico por nifurtimox Estas células son monocitos humanos derivados de leucemia monocitica aguda los cuales pueden diferenciarse a su fenotipo adherente con algunos agentes como PMA. Estas células han sido utilizadas en la prueba cometa para determinar la genotoxicidad de agentes contaminantes, micotoxinas y del trióxido de arsénico (otro inductor de ROS) entre otros (40-42). Igualmente se han utilizado en determinar el efecto protectivo del ácido gálico y de la fagocitosis de partículas de cromo y cobalto al efecto genotóxico $(43,44)$. Algunos de estos ensayos además de analizar el daño directo al ADN (o SSB roturas en una sola cadena del ADN) han determinado el daño oxidativo de ADN utilizando enzimas como la formamido-pirimidinoglicosilasa (FPG) o la endonucleasa III que reconocen purinas o pirimidinas oxidadas, aumentando la especificidad y sensibilidad del ensayo al aumentar la migración del ADN. (42). Cuando se utilizó ensayo cometa para detectar daños en el ADN por las micotoxinas en las células THP-1 no se observó aumento de daño en el ADN sin embargo este fue observado al utilizar FPG indicando el daño oxidativo (42). Sin embargo (y aunque utilizando leucocitos humanos no células THP-1) tratamiento con nifurtimox en leucocitos mostró un daño oxidativo del ADN dependiente de la dosis del compuesto donde dosis mayores de $87 \mu \mathrm{M}$ mostraron una oxidación del ADN (4). A pesar que las células THP-1 por ser células tumorales podrían ser más activas en la producción de ROS que los leucocitos normales sería interesante determinar el daño oxidativo del ADN inducido por el nifurtimox en estas células para tratar de encontrar una mayor sensibilidad. Sin embargo esta línea celular está siendo utilizada ampliamente en el ensayo cometa además como célula blanco para realizar calibraciones con muestras de referencia orientados a determinar la variación de los resultados entre los laboratorios (45). 
Un resultado interesante observado en este trabajo fueron los obtenidos con las células Vero las cuales en todos los experimentos realizados mostraron un "efecto cometa" en los controles negativos (células tratadas sin nifurtimox). Las células Vero, son células epiteliales renales obtenidas de mono utilizadas en el laboratorio para diferentes propósitos (aislamiento de virus, vacunas, pruebas de tamizaje de medicamentos contra tripanosomátidos). Un porcentaje apreciable de daño del ADN ha sido reportado en los controles negativos de ensayos cometas utilizando células Vero $(46,47)$. Consideramos que se debe ser muy cuidadoso al analizar los resultados utilizando esta línea celular.

\section{Conclusión}

En este trabajo se demostró que el nifurtimox ejerce un efecto genotóxico y citotóxico dependiente de la dosis utilizada en las líneas celulares NIH/3T3 y J774. Los porcentajes de daño del ADN en la línea de monocitos humanos THP-1 fueron menos del $15 \%$ y en las células Vero no se encontraron diferencias con los controles negativos. La extensión del daño de ADN puede ser influenciado por características de la línea celular, como los procesos de reparación del ADN, la susceptibilidad al compuesto y permeabilidad de la membrana. (48).

\section{Agradecimientos}

El presente trabajo fue financiado por el Instituto Colombiano para el Desarrollo de la Ciencia y la Tecnología "Francisco José de Caldas" COLCIENCIAS Código 1102-5192-9044 Memorando 20103600125183 y por el proyecto Código 1388 de la Vicerrectoría de Investigación y Extensión de la Universidad Industrial de Santander. AMV fue financiada por el programa de Jóvenes Investigadores convocatoria 566 convenio 0727-2012.

\section{Conflicto de intereses}

Los autores declaran no tener conflicto de intereses en la realización de este trabajo.

\section{Referencias}

1. WHO | Chagas disease (American trypanosomiasis). World Health Organization; Available from: http://www.who.int/ mediacentre/factsheets/fs340/en/

2. Docampo R MS. Biochemical toxicology of antiparasitic compounds used in the chemotherapy and chemoprophylaxis of American trypanosomiasis (Chagas' disease). Reviews in Biochemical Toxicology 1985. $159-204$

3. Raether W, Hänel H. Nitroheterocyclic drugs with broad spectrum activity. Parasitol Res. 2003 ;90 Supp 1:S19-39.

4. Buschini A, Ferrarini L, Franzoni S, Galati S, Lazzaretti M, Mussi F, et al. Genotoxicity revaluation of three commercial nitroheterocyclic drugs: nifurtimox, benznidazole, and metronidazole. J Parasitol Res 2009:463575.

5. Ohnishi T, Ohashi Y, Nozu K, Inoki S. Mutagenicity of nifurtimox in Escherichia coli. Mutat Res 1980;77(3):241-4.

6. Nagel R. Genotoxicity studies with two antichagasic drugs. Mutat Res 1987;191(1):17-20.

7. Ferreira RC, Ferreira LC. Mutagenicity of nifurtimox and benznidazole in the Salmonella/microsome assay. Brazilian J Med Biol Res 1986;19(1):19-25.
8. Nagel R, Nepomnaschy I. Mutagenicity of 2 anti-chagasic drugs and their metabolic deactivation. Mutat Res 1983;117(3-4):23742.

9. Moraga AA, Graf U. Genotoxicity testing of antiparasitic nitrofurans in the Drosophila wing somatic mutation and recombination test. Mutagenesis 1989;4(2):105-10.

10. Teixeira AR, Calixto MA, Teixeira ML. Chagas' disease: carcinogenic activity of the antitrypanosomal nitroarenes in mice. Mutat Res 1994 ;305(2):189-96.

11. Bartel LC, Montalto de Mecca M, de Castro CR, Bietto FM, Castro JA. Metabolization of nifurtimox and benznidazole in cellular fractions of rat mammary tissue. Hum Exp Toxicol 2010;29(10):813-22.

12. Buchanan-Kilbey G, Djumpah J, Papadopoulou M V, Bloomer W, Hu L, Wilkinson SR, et al. Evaluating the developmental toxicity of trypanocidal nitroaromatic compounds on zebrafish. Acta Trop 2013;128(3):701-5.

13. Iatropoulos MJ, Wang CX, von Keutz E, Williams GM. Assessment of chronic toxicity and carcinogenicity in an accelerated cancer bioassay in rats of Nifurtimox, an antitrypanosomiasis drug. Exp Toxicol Pathol Off J Gesellschaft für Toxikologische Pathol 2006 ;57(5-6):397-404.

14. Gorla NB, Ledesma OS, Barbieri GP, Larripa IB. Thirteenfold increase of chromosomal aberrations non-randomly distributed in chagasic children treated with nifurtimox. Mutat Res 1989;224(2):263-7.

15. Gorla NB, Castro JA. Micronucleus formation in bone marrow of mice treated with nifurtimox or benznidazole. Toxicol Lett 1985;25(3):259-63.

16. Ostling O, Johanson KJ. Microelectrophoretic study of radiationinduced DNA damages in individual mammalian cells. Biochem Biophys Res Commun 1984;123(1):291-8.

17. Singh NP, McCoy MT, Tice RR, Schneider EL. A simple technique for quantitation of low levels of DNA damage in individual cells. Exp Cell Res 1988;175(1):184-91.

18. Singh NP, Tice RR, Stephens RE, Schneider EL. A microgel electrophoresis technique for the direct quantitation of DNA damage and repair in individual fibroblasts cultured on microscope slides. Mutat Res 1991;252(3):289-96.

19. Collins AR. The comet assay for DNA damage and repair: principles, applications, and limitations. Mol Biotechnol 2004;26(3):249-61.

20. OECD Guidelines for the Testing of Chemicals, Section 4, Test No. 432: In Vitro 3T3 NRU Phototoxicity Test. Available from: http://www.oecd-ilibrary.org/content/book/9789264071162-en

21. Tice RR, Agurell E, Anderson D, Burlinson B, Hartmann A, Kobayashi $\mathrm{H}$, et al. Single cell gel/comet assay: guidelines for in vitro and in vivo genetic toxicology testing. Environ Mol Mutagen 2000;35(3):206-21.

22. OECD Guidelines for the Testing of Chemicals, Section 4, Test No. 489: In Vivo Mammalian Alkaline Comet Assay. Available from: http://www.oecd-ilibrary.org/environment/test-no-489-invivo-mammalian-alkaline-comet-assay_9789264224179-en

23. Mosmann T. Rapid colorimetric assay for cellular growth and survival: Application to proliferation and cytotoxicity assays. J Immunol Methods 1983;65(1-2):55-63.

24. Moktan S, Raucher D. Anticancer activity of proapoptotic peptides is highly improved by thermal targeting using elastinlike polypeptides. Int J Pept Res Ther 2012;18(3):227-37.

25. Jałoszyński P, Kujawski M, Czub-Swierczek M, Markowska J, Szyfter K. Bleomycin-induced DNA damage and its removal in lymphocytes of breast cancer patients studied by comet assay. Mutat Res 1997;385(3):223-33. 
26. Muelas S, Suárez M, Pérez R, Rodríguez H, Ochoa C, Escario JA, et al. In vitro and in vivo assays of 3,5-disubstituted-tetrahydro2H-1,3,5-thiadiazin-2-thione derivatives against Trypanosoma cruzi. Memórias do Inst Oswaldo Cruz 2002;97(2):269-72.

27. Hall BS, Bot C, Wilkinson SR. Nifurtimox activation by trypanosomal type I nitroreductases generates cytotoxic nitrile metabolites. J Biol Chem 2011 Apr 15;286(15):13088-95.

28. Khraiwesh MH, Lee CM, Brandy Y, Akinboye ES, Berhe S, Gittens G, et al. Antitrypanosomal activities and cytotoxicity of some novel imido-substituted 1,4-naphthoquinone derivatives. Arch Pharm Res 2012;35(1):27-33.

29. Leal SM, Pino N, Stashenko EE, Martínez JR, Escobar P. Antiprotozoal activity of essential oils derived from Piper spp. grown in Colombia. J Essent Oil Res 2013;25(6):512-9.

30. Saulnier Sholler GL, Bergendahl GM, Brard L, Singh AP, Heath BW, Bingham PM, et al. A phase 1 study of nifurtimox in patients with relapsed/refractory neuroblastoma. J Pediatr Hematol Oncol 2011;33(1):25-30.

31. Saulnier Sholler GL, Brard L, Straub JA, Dorf L, Illeyne S, Koto K, et al. Nifurtimox induces apoptosis of neuroblastoma cells in vitro and in vivo. J Pediatr Hematol Oncol 2009;31(3):187-93.

32. Kelly JM, Taylor MC, Smith K, Hunter KJ, Fairlamb AH. Phenotype of recombinant Leishmania donovani and Trypanosoma cruzi which over-express trypanothione reductase. Sensitivity towards agents that are thought to induce oxidative stress. Eur J Biochem 1993;218(1):29-37.

33. Flint MS, Baum A, Episcopo B, Knickelbein KZ, Liegey Dougall $\mathrm{AJ}$, Chambers WH, et al. Chronic exposure to stress hormones promotes transformation and tumorigenicity of 3T3 mouse fibroblasts. Stress 2013;16(1):114-21.

34. Demir E, Akça H, Kaya B, Burgucu D, Tokgün O, Turna F, et al. Zinc oxide nanoparticles: genotoxicity, interactions with UV-light and cell-transforming potential. J Hazard Mater 2014;264:420-9.

35. Demir E, Akça H, Turna F, Aksakal S, Burgucu D, Kaya B, et al. Genotoxic and cell-transforming effects of titanium dioxide nanoparticles. Environ Res 2015;136:300-8.

36. Bittencourt LS, Machado DC, Machado MM, Dos Santos GFF, Algarve TD, Marinowic DR, et al. The protective effects of guaraná extract (Paullinia cupana) on fibroblast NIH-3T3 cells exposed to sodium nitroprusside. Food Chem Toxicol 2013;53:119-25.

37. Wu Y, Qi X, Gong L, Xing G, Chen M, Miao L, et al. Identification of BC005512 as a DNA damage responsive murine endogenous retrovirus of GLN family involved in cell growth regulation. PLoS One 2012;7(4):e35010.

38. Cappoen D, Majce V, Uythethofken C, Urankar D, Mathys V, Kočevar M, et al. Biological evaluation of diazene derivatives as anti-tubercular compounds. Eur J Med Chem 2014;74:85-94.

39. Claes P, Cappoen D, Uythethofken C, Jacobs J, Mertens B, Mathys $\mathrm{V}$, et al. 2,4-Dialkyl-8,9,10,11-tetrahydrobenzo[g]pyrimido[4,5-c] isoquinoline-1,3,7,12(2H,4H)-tetraones as new leads against Mycobacterium tuberculosis. Eur J Med Chem 2014;77:409-21.

40. Don Porto Carero A, Hoet PH, Verschaeve L, Schoeters G, Nemery B. Genotoxic effects of carbon black particles, diesel exhaust particles, and urban air particulates and their extracts on a human alveolar epithelial cell line (A549) and a human monocytic cell line (THP-1). Environ Mol Mutagen 2001;37(2):155-63.

41. Graham-Evans B, Cohly HHP, Yu H, Tchounwou PB. Arsenicinduced genotoxic and cytotoxic effects in human keratinocytes, melanocytes and dendritic cells. Int J Environ Res Public Health 2004;1(2):83-9.
42. Rakkestad KE, Skaar I, Ansteinsson VE, Solhaug A, Holme JA, Pestka JJ, et al. DNA damage and DNA damage responses in THP-1 monocytes after exposure to spores of either Stachybotrys chartarum or Aspergillus versicolor or to T-2 toxin. Toxicol Sci 2010;115(1):140-55.

43. Papageorgiou I, Shadrick V, Davis S, Hails L, Schins R, Newson R, et al. Macrophages detoxify the genotoxic and cytotoxic effects of surgical cobalt chrome alloy particles but not quartz particles on human cells in vitro. Mutat Res 2008;643(1-2):11-9.

44. Kuppan G, Balasubramanyam J, Monickaraj F, Srinivasan G, Mohan V, Balasubramanyam M. Transcriptional regulation of cytokines and oxidative stress by gallic acid in human THP-1 monocytes. Cytokine 2010;49(2):229-34.

45. Forchhammer L, Johansson C, Loft S, Möller L, Godschalk RWL, Langie SAS, et al. Variation in the measurement of DNA damage by comet assay measured by the ECVAG inter-laboratory validation trial. Mutagenesis 2010;25(2):113-23.

46. Rjiba-Touati K, Ayed-Boussema I, Belarbia A, Azzebi A, Achour A, Bacha H. Protective effect of recombinant human erythropoeitin against cisplatin cytotoxicity and genotoxicity in cultured Vero cells. Exp Toxicol Pathol Off J Gesellschaft für Toxikologische Pathol 2013;65(1-2):181-7.

47. Mihai CT, Rotinberg P, Brinza F, Vochita G. Extremely lowfrequency electromagnetic fields cause DNA strand breaks in normal cells. J Environ Heal Sci Eng 2014;12(1):15.

48. Andrighetti-Fröhner CR, Kratz JM, Antonio R V, Creczynski-Pasa TB, Barardi CRM, Simões CMO. In vitro testing for genotoxicity of violacein assessed by Comet and Micronucleus assays. Mutat Res 2006;603(1):97-103. 